\title{
Pharmaceutical and Herbal Fingerprinting by Means of Chromatographic Techniques
}

\author{
Teresa Kowalska, ${ }^{1}$ Hassan Y. Aboul-Enein, ${ }^{2}$ Yvan Vander Heyden, ${ }^{3}$ \\ Irena Vovk, ${ }^{4}$ and Monika Waksmundzka-Hajnos ${ }^{5}$ \\ ${ }^{1}$ Institute of Chemistry, University of Silesia, 9 Szkolna Street, 40-006 Katowice, Poland \\ ${ }^{2}$ Department of Pharmaceutical and Medicinal Chemistry, National Research Centre, Dokki, Cairo 12311, Egypt \\ ${ }^{3}$ Department of Analytical Chemistry and Pharmaceutical Technology (FABI), Vrije Universiteit Brussel, Laarbeeklaan 103, \\ 1090 Brussels, Belgium \\ ${ }^{4}$ Laboratory for Food Chemistry, National Institute of Chemistry, Hajdrihova 19, 1000 Ljubljana, Slovenia \\ ${ }^{5}$ Departament of Inorganic Chemistry, Medical University of Lublin, 6 Chodźki Street, 20-093 Lublin, Poland
}

Correspondence should be addressed to Teresa Kowalska, teresa.kowalska@us.edu.pl

Received 15 November 2011; Accepted 15 November 2011

Copyright (C) 2012 Teresa Kowalska et al. This is an open access article distributed under the Creative Commons Attribution License, which permits unrestricted use, distribution, and reproduction in any medium, provided the original work is properly cited.

In spite of a continuous and considerable progress in the performance of each chromatographic technique, separation, identification, and quantification of complex mixtures still remain a challenging analytical task. There are multiple bottlenecks on the way to complete fulfillment of this task, basically depending on the origin and the chemical nature of a given mixture. With all pharmaceutical and herbal mixtures-equally those of known and unknown originsa similar major problem consists in their largely unpredictable composition. Among the most sensitive analytical areas, chromatographic characterization of pharmaceutical preparations and herbal materials ought to be named.

The quality control of pharmaceutical preparations poses very strict demands not only on monitoring of active ingredients, but also of the excipients, and the impurities contained therein. In recent years, the pharmaceutical markets worldwide have been heavily invaded by counterfeit products, an acute problem which in turn rises the necessity to develop rapid yet reliable chromatographic procedures of distinguishing between the legal and the illegal products. A similar problem exists with medicinal plants, which in recent years started attracting the growing interest both from the side of the learned medical circles and the patients. The complex and unpredictable chemical composition of plant material and the high costs of phytochemical standards virtually forced analysts to look for simple chromatographic procedures which might allow rapid comparison among the different batches of a given plant. As plants are traded mostly in a crumbled or granulated form, their botanical morphology is largely destroyed, which in turn generates a possibility of an easy falsification. This is one more serious reason to look for rapid and reliable chromatographic procedures enabling identification of botanical species.

The paper by $\mathrm{H}$. Tanaka et al. describes application of an advanced separation technique analogous to that known from molecular biology as Western blotting and enabling the detection of specific proteins in given samples of tissue homogenates or extracts. The authors thoroughly describe the modification to this technique, known as Eastern blotting (basically used for the analysis of the carbohydrate epitopes), to fingerprinting of solasodine glycosides and ginsenosides contained in Solanum and Panax ginseng species, respectively, that is, in traditional Chinese herbal medicines.

The paper by $€$. Cieśla in this special issue provides an upto-the-date overview of biological fingerprinting of herbal samples by means of liquid chromatography (HPLC and TLC). Biological fingerprinting is a relatively new concept in the quality control of botanical material, offering unique and very promising solutions which should draw even more attention in the future. The basic principle of biological fingerprinting consists in chromatographic highlighting of the biological role of certain chromatographically separated fractions or individual chemical species, in contrast with the biologically irrelevant components of the same mixtures. 
The paper by $€$. Komsta also is a review paper, focusing on the applications of chemometrics to fingerprinting in TLC. Due to the fact that the TLC fingerprinting not always is fully instrumented (i.e., it is not always based on densitometric detection, but often limited to the videoscan evidence alone), chemometric evaluation of the thin-layer chromatographic fingerprints is less frequent than that of the high-performance liquid chromatograms. The author of this paper provides a complete overview of the literature on applications of chemometrics to the thin-layer chromatographic fingerprints, emphasizing advantages of these applications and also their technique-related specificity.

In the paper by Z. Hua-Bin et al., the readers are introduced to the modern quality control methodology applied to traditional Chinese medicines (TCMs) of herbal origin. This quality control methodology has been elaborated mostly by Chinese research teams and many papers reviewed in this article have originally appeared in Chinese (which is an evident advantage of the actually presented resume). The described quality control methodology reaches from the simple visual inspection and comparison of the different fingerprints to more advanced approaches, such as clustering analysis, principal component analysis (PCA), and similarity analysis.

In the paper by C. A. Diagone et al., the authors employ the classical HPLC fingerprinting approach with the UV and PDA detection systems to the fingerprinting of the flavonoid profiles derived from two plants well established in the Brazilian folk medicine, that is, Maytenus aquifolium and Maytenus ilicifolia. The obtained results are compared with those originating from capillary zone electrophoresis (CZE) and the advantages of the latter technique are highlighted. A conclusion is drawn about the applicability of CZE in the routine quality control of the two aforementioned Brazilian phytopharmaceuticals.

The paper by J. Rzepa et al. handles the methodical aspects of fingerprinting the volatile fraction of botanical origin. As case study, it focuses on the composition of the volatile fraction in winter savory (Satureja montana), an important culinary herb widespread in the Mediterranean zone and in the Balkans, and having a firm position in the traditional European medicine of these regions. The authors demonstrate the superiority of the fingerprinting results originating from the headspace gas chromatography with mass spectrometric detection (HS-GC/MS) over those obtained using the recommended European and Polish pharmacopoeial hydrodistillation techniques, followed by GC/MS fingerprinting.

The paper by D. L. Chothani et al. focuses on the thinlayer chromatographic fingerprinting, isolation, and quantification of a marker compound contained in Ruellia tuberosa, the medicinal herb acknowledged by the traditional Indian pharmacognosy. The most valuable features of this study are that it offers an efficient thin-layer chromatographic fingerprinting procedure (which is relatively less common than the usage of the fully instrumented chromatographic techniques), and the isolation, identification, and quantification of the marker compound prior to its identification. The authors plan to carry out identification of this marker compound in the forthcoming steps of their study, again with a substantial contribution of planar chromatography.

The paper by M. Sajewicz et al. furnishes a good practical example of the application of chemometrics to fingerprinting of the flavonoid fraction derived from the selected sage (Salvia) species and analyzed by means of HPLC/DAD. Chemometric purification of the chromatograms is presented as an inevitable pretreatment step, followed by grouping the purified chromatographic fingerprints by means of PCA.

Teresa Kowalska

Hassan Y. Aboul-Enein Yvan Vander Heyden Irena Vovk Monika Waksmundzka-Hajnos 


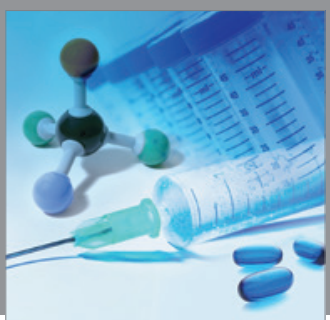

International Journal of

Medicinal Chemistry

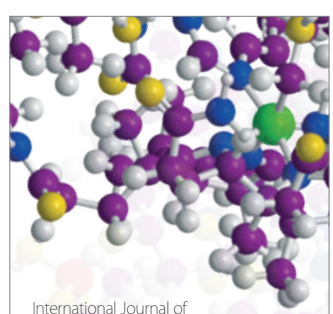

Carbohydrate Chemistry

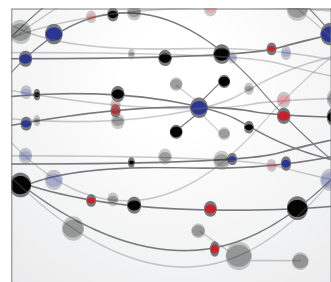

The Scientific World Journal
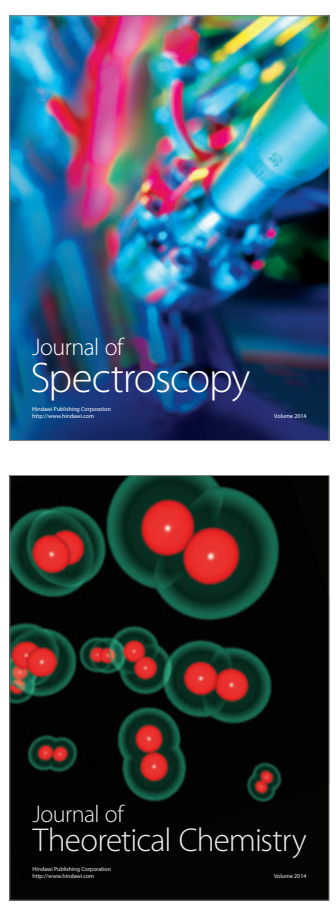
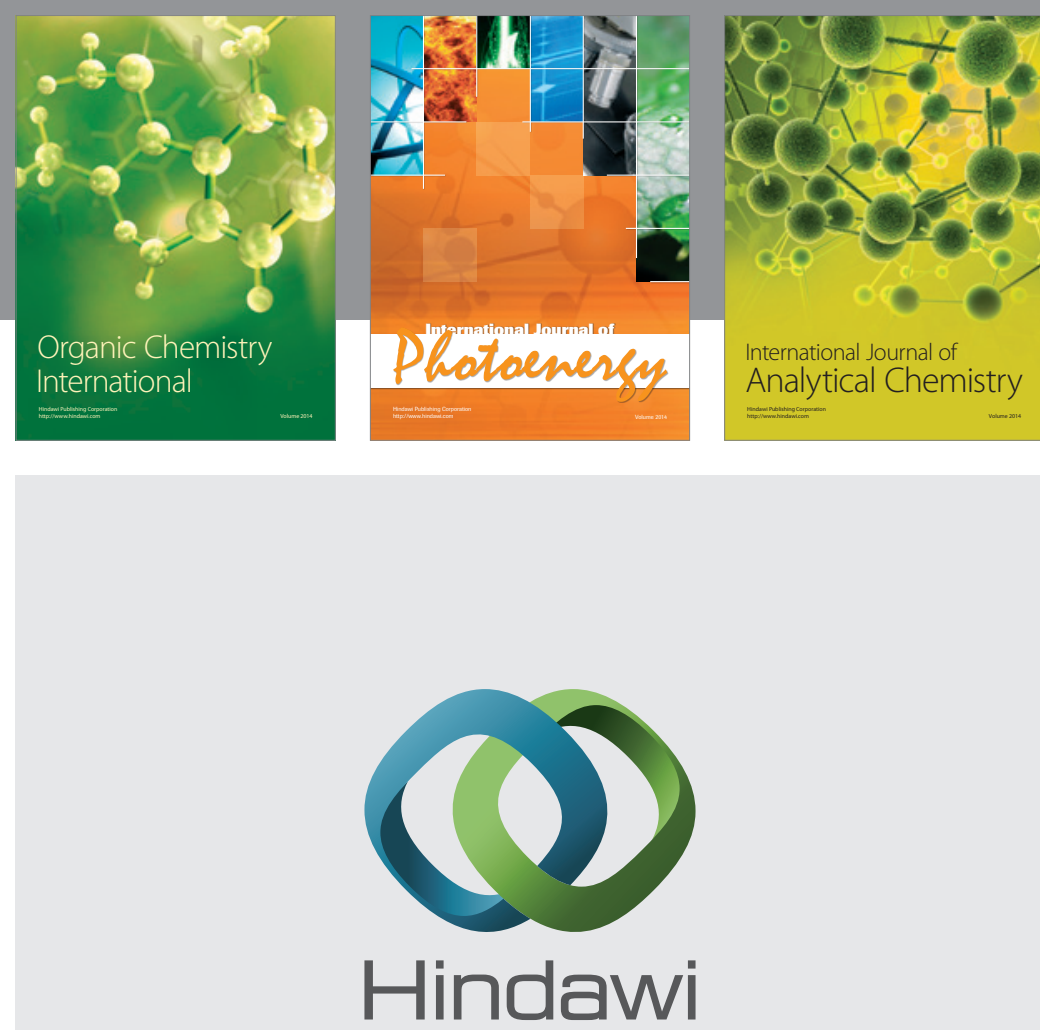

Submit your manuscripts at

http://www.hindawi.com
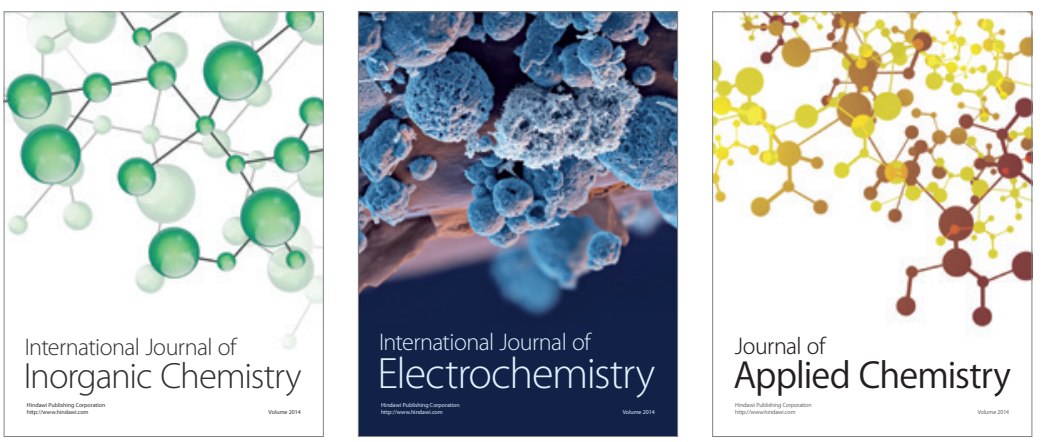

Journal of

Applied Chemistry
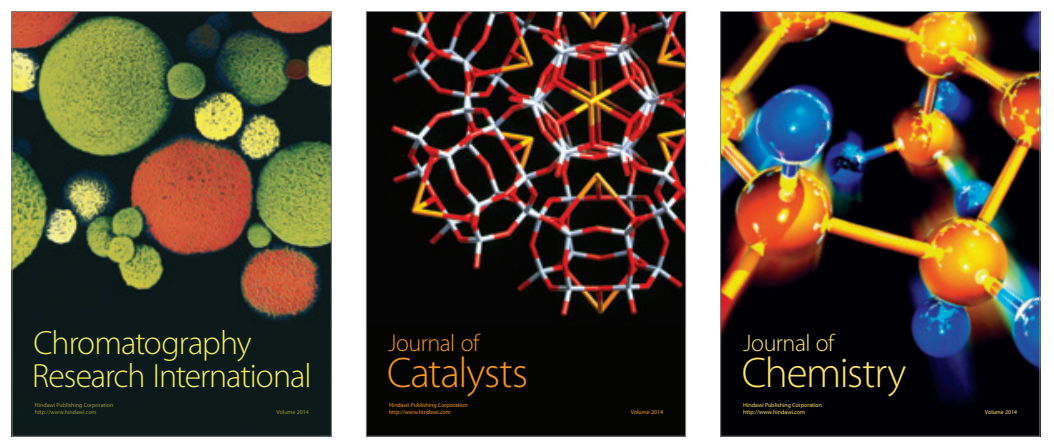
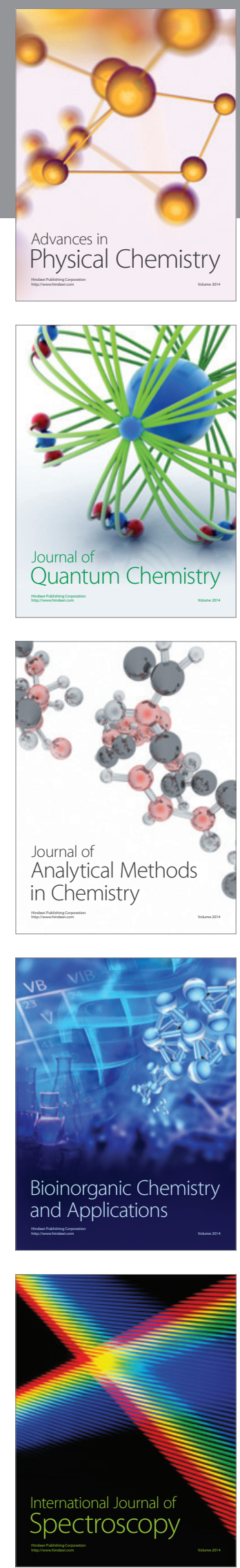